\title{
Clindamycin-Induced Acute Kidney Injury: Large Biopsy Case Series
}

\author{
Honglang Xie Huiping Chen Yangling Hu Shutian Xu Qunpeng He Jing Liu \\ Weixin Hu Zhihong Liu \\ Research Institute of Nephrology, Jinling Hospital, Nanjing University Clinical School of Medicine, Nanjing, PR China
}

\section{Key Words}

Clindamycin $\cdot$ Kidney $\cdot$ Injury $\cdot$ Biopsy

\begin{abstract}
Background: While clindamycin-induced acute kidney injury $(\mathrm{AKI})$ is uncommon, it has occurred more frequently in recent years. Summary: We investigated 24 patients diagnosed with clindamycin-induced AKI retrospectively. The dosage of clindamycin was 1.0-1.5 g/day. Fifteen patients had episodes of gross hematuria, but fever, skin rash and eosinophilia were rare. Urine analysis revealed mild proteinuria and severe tubular dysfunction. Twenty-three patients were diagnosed with AKI stage 3 upon admission. The clindamycin lymphocyte transformation assay was positive for $63.2 \%$ of the patients. Acute interstitial nephritis (AIN) and acute tubular necrosis (ATN) were proven by renal biopsy, and renal insufficiency appeared to result from tubular toxicity and drug crystals. In the majority (87.5\%) of the patients, AKI was severe and required renal replacement therapy, but all of their renal function recovered significantly 2 months after discharge. Clindamycin-induced AKI is largely reversible and has episodes of gross hematuria. Renal biopsies confirmed AIN or ATN in these patients.

Copyright $\odot 2013$ S. Karger AG, Basel
\end{abstract}

\section{Introduction}

Acute kidney injury (AKI) is a broad clinical syndrome defined by an abrupt decrease in kidney function within $48 \mathrm{~h}$ caused by various etiologies [1], and in such a condition, even a small alteration in serum creatinine (SCr) indicates poor outcomes. The incidence of AKI has increased dramatically in recent years [2-4]. According to Uchino et al. [5], drugs are common causes of AKI and account for nearly $20 \%$ of all cases of AKI in the intensive care unit, especially nonsteroidal anti-inflammatory drugs and antibiotics. Clindamycin is a commonly used antibiotic with broad antimicrobial activity, but the frequency of reported side effects has risen recently in China. The present paper reports on biopsy-based case series of clindamycin-induced AKI.

\section{Materials and Methods}

We retrospectively reviewed 46 patients exposed to clindamycin presenting at the Institute of Nephrology in Jinling Hospital from August 2008 to June 2012. Twenty-four patients were found to have clindamycin-induced AKI. Twenty-two patients were excluded because they were receiving a combination therapy with other suspected nephrotoxic drugs (ribavirin, nonsteroidal antiinflammatory drugs, aminoglycosides, acyclovir), had a history of 
chronic kidney disease or were without renal biopsy result. All 24 patients included in this report met the following criteria: (1) no previous history of underlying chronic kidney disease, (2) met the Acute Kidney Injury Network (AKIN) criteria of AKI within $72 \mathrm{~h}$ after the infusion of clindamycin and (3) no obvious alternative causes of AKI (e.g. volume insufficiency, septic shock, urinary obstruction or hemorrhagic fever with renal syndrome).

The clinical and biological characteristics of the patients at the time of AKI diagnosis are summarized in tables 1-3 and figure 1. AKI staging was performed according to the AKIN SCr criteria. Hypertension was defined as systolic blood pressure $\geq 140 \mathrm{~mm} \mathrm{Hg}$ and/or diastolic blood pressure $\geq 90 \mathrm{~mm} \mathrm{Hg}$. Urinalysis was performed and urine markers like $\mathrm{N}$-acetyl- $\beta$-D-glucosaminidase, retinol-binding protein, lysozyme, cystatin $\mathrm{C}$, kidney injury molecule-1, neutrophil gelatinase-associated lipocalin and interleukin-18 were measured on admission to detect the dysfunction of renal tubular preliminarily. Anemia was diagnosed as hemoglobin levels $\leq 12 \mathrm{~g} / \mathrm{dl}$ in males and $\leq 11 \mathrm{~g} / \mathrm{dl}$ in females.

Lymphocyte transformation tests were performed in 19 patients and 19 healthy individuals without any allergic history. Peripheral blood mononuclear cells were isolated and cultured with clindamycin at various concentrations. After 6 days, $0.5 \mu \mathrm{Ci}$ of ${ }^{3} \mathrm{H}$ thymidine was added for $8-14 \mathrm{~h}$. The cells were harvested, and the incorporated radioactivity was measured with a $\beta$-counter as an indicator of proliferation [6].

Renal biopsies were performed in all 24 patients. The specimens were formalin fixed (10\%) and paraffin imbedded; $1.5-\mu \mathrm{m}$ paraffin sections were stained with hematoxylin-eosin, periodic acid-Schiff, periodic acid-silver methenamine and Masson's trichrome stain. For each sample, $4-\mu \mathrm{m}$ cryostat tissue sections were stained with an immunofluorescence panel including IgG, IgA, IgM, C3, C1q and fibrin. Staining for CD4, CD8 and CD68 was also performed. Acute interstitial nephritis (AIN) was characterized by the presence of marked inflammatory infiltrates and edema within the interstitium. Acute tubular necrosis (ATN) was characterized by tubular degeneration, regeneration and interstitial edema, without massive cell infiltrates and tubulitis. The glomeruli were normal in both ATN and AIN. The severity of the interstitial infiltrate was scored as mild, moderate or severe. All biopsies were analyzed by at least two pathologists with a special interest in renal pathology.

Descriptive statistics $(\mathrm{x} \pm \mathrm{SD})$ were used to summarize and describe the quantitative characteristics of the studies.

\section{Results}

The cohort of 24 patients had a male:female ratio of 14:10. The mean age at renal biopsy was $42.1 \pm 12.5$ years (range: 20-60). Upper respiratory infection (45.8\%) and gastroenteritis $(16.7 \%)$ were the most common reasons for clindamycin therapy (table 1).

The common dose of clindamycin in these patients was $0.5-0.75 \mathrm{~g}$, intravenous infusion twice daily. The average onset of AKI occurred 1 day after the first dosage (range: 0.5 h to 4 days). On admission, only 1 patient was classified as AKI stage 1 and 23 patients (95.8\%) were classified as AKI stage 3. Symptoms and their frequency
Table 1. Reasons for clindamycin treatment

\begin{tabular}{lc}
\hline Diagnosis & Number of patients (\%) \\
\hline URI/COPD & $11(45.8)$ \\
Gastroenteritis & $4(16.7)$ \\
Toothache & $2(8.3)$ \\
Prevention of infection & \\
$\quad$ after aseptic operation & $2(8.3)$ \\
Trauma & $2(8.3)$ \\
Panaritium & $1(4.2)$ \\
Cholecystitis & $1(4.2)$ \\
Arthralgia & $1(4.2)$ \\
\hline
\end{tabular}

COPD = Chronic obstructive pulmonary disease; $\mathrm{URI}=$ upper respiratory infection.

Table 2. Laboratory examination of AKI associated with clindamycin on admission

\begin{tabular}{lllc}
\hline Parameter & $\begin{array}{l}\text { Normal } \\
\text { range }\end{array}$ & $\begin{array}{l}\text { Mean } \\
(\mathrm{x} \pm \mathrm{SD})\end{array}$ & Range \\
\hline Blood & & & \\
Hb, g/dl & & & \\
$\quad$ Female & $11.0-15.0$ & $11.5 \pm 1.5$ & $8.1-14.3$ \\
$\quad$ Male & $12.0-16.0$ & & \\
Eosinophils, \% & $<7.0$ & $1.3 \pm 1.2$ & $0-4.0$ \\
SCr, mg/dl & $0.51-1.24$ & $7.8 \pm 3.4$ & $1.6-14.4$ \\
Cystatin C, mg/dl & $0.45-1.18$ & $3.0 \pm 1.2$ & $1.5-4.9$ \\
\hline Urine & & & \\
Proteinuria, g/24h & $\leq 0.4$ & $0.5 \pm 0.4$ & $0.1-1.5$ \\
NAG, $\mu / g \cdot c r$ & 16.5 & $48.3 \pm 33.9$ & $10.7-151.9$ \\
RBP, mg/l & $\leq 0.5$ & $11.5 \pm 15.1$ & $0.4-44.4$ \\
Lysozyme, mg/l & $<1.0$ & $4.5 \pm 6.5$ & $0.5-23.3$ \\
Cystatin C, mg/l & $<0.15$ & $0.9 \pm 1.2$ & $0.02-3.7$ \\
NGAL, $\mu \mathrm{g} / \mathrm{l}$ & $<20$ & $427.3 \pm 1,179.2$ & $12.5-4,758.6$ \\
KIM-1, $\mu$ g/l & $<0.4$ & $2.3 \pm 6.3$ & $0.2-26.5$ \\
IL-18, ng/l & $<20$ & $34.8 \pm 43.1$ & $0.5-150.1$ \\
\hline
\end{tabular}

$\mathrm{NAG}=\mathrm{N}$-acetyl- $\beta$-D-glucosaminidase; $\mathrm{RBP}=$ retinol-binding protein; NGAL = neutrophil gelatinase-associated lipocalin; KIM1 = kidney injury molecule-1; IL-18 = interleukin-18.

Table 3. Kidney interstitial cell infiltration in 8 patients

\begin{tabular}{llc}
\hline Cell phenotype & Normal range & Mean $(\mathrm{x} \pm \mathrm{SD})$ \\
\hline $\mathrm{CD} 4+, / \mathrm{mm}^{2}$ & $27.2 \pm 11.3$ & $192 \pm 166.8$ \\
$\mathrm{CD} 8+, / \mathrm{mm}^{2}$ & $20.8 \pm 7.3$ & $168.6 \pm 152.9$ \\
$\mathrm{CD} 4+, / \mathrm{CD} 8+$ & $1.29 \pm 1.26$ & $1.1 \pm 0.2$ \\
$\mathrm{CD} 68+, / \mathrm{mm}^{2}$ & negative & $460 \pm 399.4$ \\
\hline
\end{tabular}




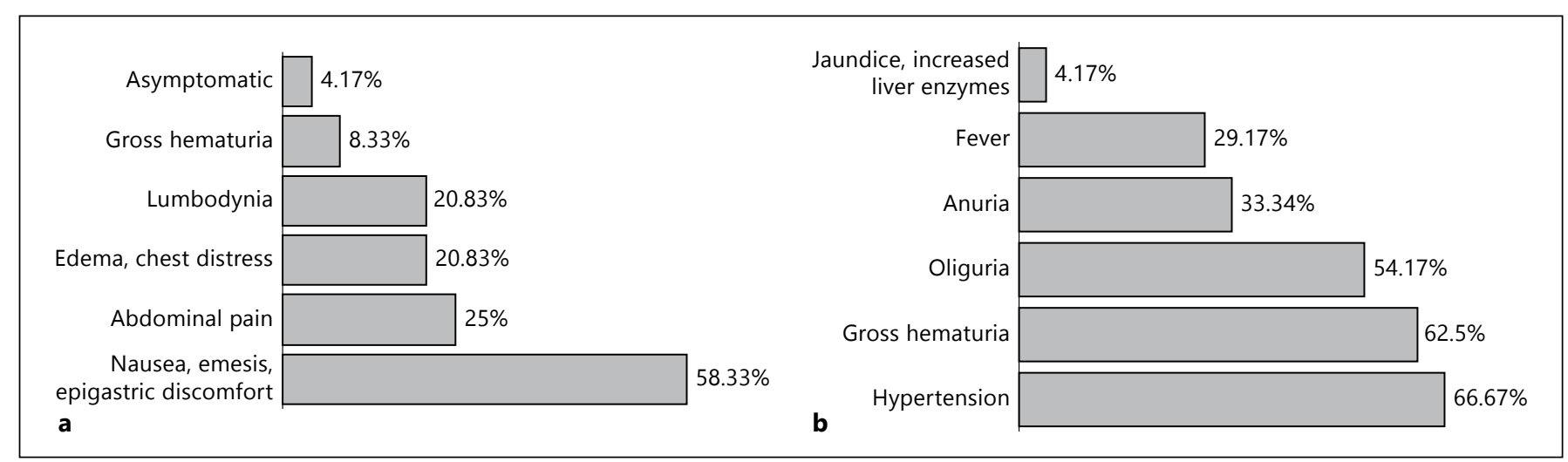

Fig. 1. Presenting symptoms (a) and clinical manifestation (b) at the time of AKI diagnosis.

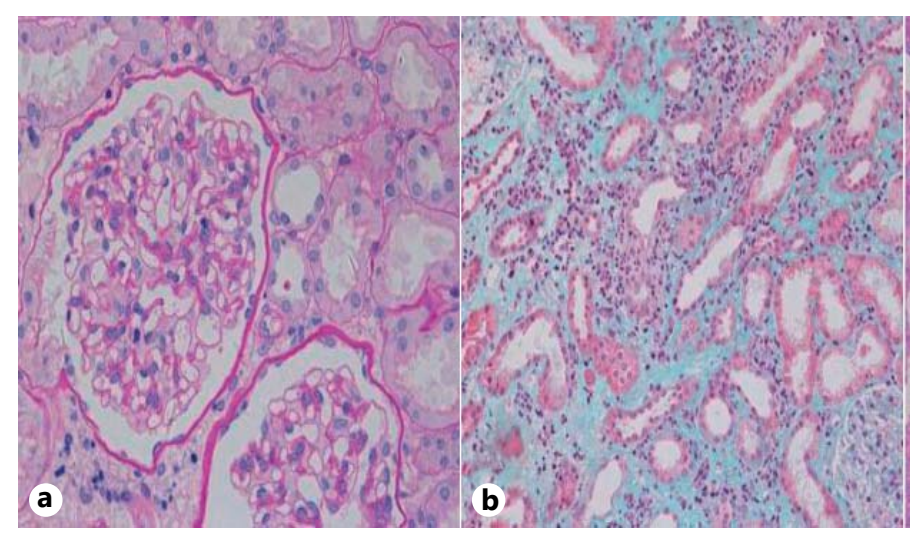

FIg. 2. a The glomeruli were unremarkable (PAS staining, $\times 400$ magnification). b The interstitium was mildly edematous with massive aggregates of inflammatory cells (Masson's trichrome staining, $\times 400$

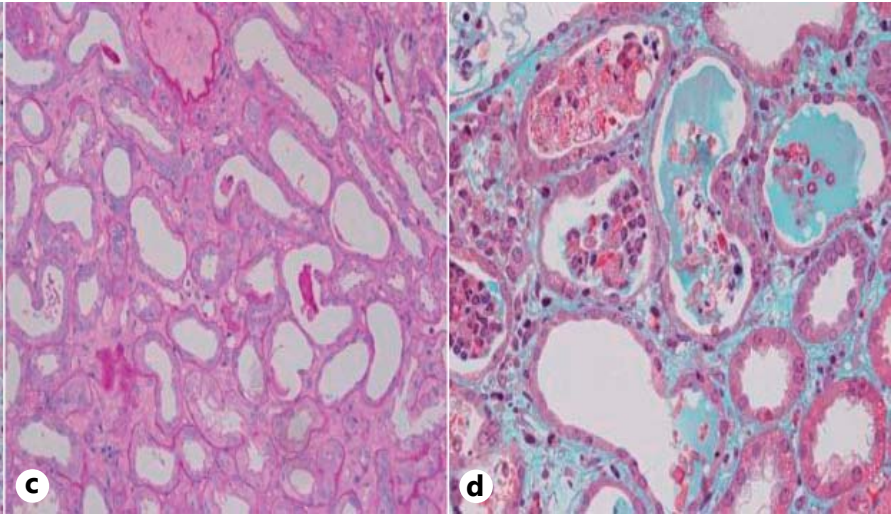

magnification). c Focal ablation of the renal epithelial brush border (PAS staining, $\times 200$ magnification). d Cellular cast in the renal tubular lumen (Masson's trichrome staining, $\times 400$ magnification). are presented in figure 1. Twelve patients (50\%) were anemic, but none of them presented with eosinophilia. Urine analysis revealed mild proteinuria $(0.5 \pm 0.4 \mathrm{~g} /$ $24 \mathrm{~h})$. Urinary sediment analysis disclosed intact red blood cells in 3 patients $\left(3.6-125 \times 10^{6} / \mathrm{ml}\right.$, normal range: $\left.<0.01 \times 10^{6} / \mathrm{ml}\right)$, dysmorphic red blood cells in 1 patient $\left(0.9 \times 10^{6} / \mathrm{ml}\right)$ and eosinophiluria in 1 patient (table 2). Renal tubular function was compromised significantly, characterized by elevated levels of $\mathrm{N}$-acetyl$\beta$-D-glucosaminidase, retinol-binding protein, lysozyme, cystatin C, kidney injury molecule-1, neutrophil gelatinase-associated lipocalin and interleukin-18. The lymphocyte transformation test was positive for 12 of 19 patients $(63.2 \%)$.

Renal biopsies were performed at the polyuric stage (19.7 \pm 12.5 days after the first dosage of clindamycin).
No immune deposits were detected on immunofluorescence study. On light microscopy, unremarkable glomerular lesions (fig. 2a) but intense interstitial infiltration of inflammatory cells (including monocytes, neutrophils, eosinophils and plasma cells) were noticed in some patients (fig. 2b). ATN was found in all specimens (fig. 2c). Renal tubulitis was identified in 19 (79.2\%) of the patients and renal epithelial cells, neutrophils, dysmorphic red blood cells and occasionally eosinophils were detected in the renal tubular lumen (fig. 2d). No granuloma was detected. The arteries and arterioles were unremarkable. In 5 patients, crystals were visualized in the tubular lumen or in the tubular epithelial cell cytoplasm, and interstitial bleeding was observed in only 1 case. Immunohistochemistry confirmed an increased number of CD4+, CD8+ and CD68+ cells in the interstitial infiltrations (table 3). Eigh- 
teen patients (75\%) were diagnosed with AIN and 6 patients (25\%) were diagnosed with ATN.

Nineteen patients $(79.2 \%)$ received oral prednisone (30 mg/day). Continuous renal replacement therapy was performed in 18 patients (75\%) and 3 patients (12.5\%) were dialyzed intermittently. All the patients discontinued renal replacement therapy, and 16 of them were discharged with normal SCr $(<1.24 \mathrm{mg} / \mathrm{dl})$. All renal indices returned to normal 2 months after discharge.

\section{Discussion}

Clindamycin, a semisynthetic chemical derivative of lincomycin, was first synthesized by Magerlein and coworkers in 1966. It is active against aerobic Gram-positive, anaerobic Gram-positive and Gram-negative bacteria, and is widely used in patients with an allergic history to antibiotics [7]. Common adverse drug reactions (ADRs) associated with clindamycin therapy include phlebitis, anaphylaxis (e.g. fever, skin rash, photosensitivity and serum sickness), neutrocytopenia, eosinophilia, vomiting, nausea, hepatotoxicity and headache; however, elevated SCr and blood urea nitrogen have rarely been reported $[8,9]$.

The incidence of ADRs associated with clindamycin has been increasing recently in China, frequently presenting as hematuria and AKI. Wu [10] studied a group of 419 ADR cases associated with clindamycin from January 2007 to November 2008 in Ningbo, including 2 cases $(0.4 \%)$ of hematuria and 54 cases $(21.9 \%)$ of acute renal failure. He [11] reported 108 ADR cases associated with clindamycin from 2005 to 2009, including hematuria and acute renal failure in 5 cases (3.5\%). Fan et al. [12] searched the China Hospital Knowledge Database (CHKD; 20032008) using 'clindamycin' as a keyword and analyzed 107 studies in total: 29 (19.9\%) patients showed kidney involvement, including 22 cases of hematuria and 7 cases of acute renal failure. Apparently, the incidence of renal injury associated with clindamycin in China cannot be ignored.

We report on biopsy-proven AKI associated with clindamycin, excluding the influence of other renal toxic drugs and history of chronic kidney disease. These patients presented with high incidences of anemia, hypertension and gross hematuria rather than the common manifestation of drug-induced AIN-like fever, skin rash and eosinophilia. Ninety-six percent of them fulfilled the criteria of AKI stage 3. The elevation of sensitive urinary markers demonstrated tubulointerstitial defect. Renal biopsies confirmed ATN and AIN. Although short-term follow-up indicated relatively good outcomes, the longterm prognosis remains to be observed.

The mechanism of drug-induced AKI is variable and includes ATN (e.g. aminoglycosides), AIN (e.g. ampicillin), hemodynamic perturbations (e.g. nonsteroidal antiinflammatory drugs and angiotensin-converting enzyme inhibitors) and tubular obstruction due to drug crystallization (e.g. acyclovir) [13]. Clindamycin is extensively metabolized in the liver, most likely by the cytochrome P450 isoenzyme 3A4, but $10 \%$ of the drug is excreted by the kidney. A high urine concentration of clindamycin may precipitate in the renal tubular lumen and lead to tubular obstruction. Renal biopsies proved the presence of crystals in the tubular lumen or in tubular epithelial cell cytoplasm in 5 patients and interstitial bleeding in 1 patient. Hematuria, especially gross hematuria, is believed to be secondary to drug crystallization, which causes tubular damage and renal function impairment [14].

From the time range of the occurrence of AKI (average 1 day, at least $0.5 \mathrm{~h}$ ), we suggest that the main pathophysiological mechanism might be direct tubular toxicity, which still calls for further studies. Tubular obstruction caused by crystals could increase the incidence of ATN. The pathogenesis of AIN is an immunologically induced hypersensitivity reaction to antigens like tubular basement membrane components, secreted tubular proteins and nonrenal proteins, and cell-mediated immunity plays a major role in its development [15]. This is reinforced by the fact that the interstitial infiltrates contained numerous mononuclear cells and immunofluorescence was negative in the renal tubular basement membrane. The lymphocyte transformation test was positive in $63.2 \%$ of the cases, which means clindamycin-specific $\mathrm{T}$ cells can be found in the peripheral blood and may release inflammatory cytokines. All of the characteristics mentioned above provide evidence that clindamycin (or some ingredient in the drug) induces renal injury mainly by damaging the renal tubule directly and stimulating the primed lymphocyte transformation.

The incidence of clindamycin-induced ADRs in China is much higher than that in other countries. Mazur et al. [16] reported that the incidence of clindamycin-induced erythema decreased from $10 \%$ in the 1970s [17] to less than $0.4 \%$ [16] at the end of the last century, and they suggested that the allergic reaction to clindamycin was very rare due to improved drug quality. However, clindamycin-induced AKI was more prevalent in China during the same period. This might be explained by the difference in the drug impurity limitation standard. The impurity limitation of clindamycin in China is $<8 \%$, while it is $<6 \%$ in the USA. 
In summary, we present a renal biopsy series of 24 patients with clindamycin nephrotoxicity. The clinical findings were characterized by transient gross hematuria and oliguric AKI. Renal tubular function was impaired severely, and the lymphocyte transformation test was positive in $63.2 \%$ of the patients. The renal pathological diagnosis indicated AIN and ATN. Despite the presence of severe renal failure, the outcomes were quite good. Clindamycin-induced AKI should not be overlooked after discontinuing the use of the offending drug since it potentially causes serious complications.

\section{Acknowledgements}

This study was supported by the key projects in the National Science and Technology Pillar Program in the Twelfth Five-Year Plan Period (grant No. 2011BAI10B07).

\section{Disclosure Statement}

No conflict of interest statement.

\section{References}

1 Mehta RL, Kellum JA, Shah SV: Acute Kidney Injury Network: report of an initiative to improve outcomes in acute kidney injury. Crit Care 2007;11:R31.

2 Ali T, Khan I, Simpson W: Incidence and outcomes in acute kidney injury: a comprehensive population-based study. J Am Soc Nephrol 2007;18:1292-1298.

>3 Nash K, Hafeez A, Hou S: Hospital-acquired renal insufficiency. Am J Kidney Dis 2002;39: 930-936.

-4 Srisawat N, Hoste E, Kellum J: Modern classification of acute kidney injury. Blood Purif 2010;29:300-307.

5 Uchino S, Kellum JA, Bellomo R: Acute renal failure in critically ill patients: a multinational, multicenter study. JAMA 2005;294:813818.
6 Kano Y, Hirahara K, Mitsuyama Y: Utility of the lymphocyte transformation test in the diagnosis of drug sensitivity: dependence on its timing and the type of drug eruption. Allergy 2007;62:1439-1444.

7 Lammintausta K, Tokola R, Kalimo K: Cutaneous adverse reactions to clindamycin: results of skin tests and oral exposure. Br J Dermatol 2002;146:643-658.

8 Sanford JP: The Sanford Guide to Antimicrobial Therapy 2011-2012, ed 41. Sperryville, Antimicrobial Therapy Inc, 2011, pp 87-88.

9 Seitz CS, Bröcker EB, Trautmann A: Allergy diagnostic testing in clindamycin-induced skin reactions. Int Arch Allergy Immunol 2009; 149:246-250.

10 Wu HW: Adverse effects of clindamycin: 419 cases report. Chin J Drug Abuse Prev Treat 2010;16:51-54.

$11 \mathrm{He}$ D: Adverse effects of clindamycin: 108 cases report. Anti-Infect Pharm 2010;7:141-142.
12 Fan M, Peng WY, Shen CY: Document analysis of the adverse effects of clindamycin. Herald Med 2010;29:963-965.

13 Dennen P, Douglas IS, Anderson R: Acute kidney injury in the intensive care unit: an update and primer for the intensivist. Crit Care Med 2010;38:261-275.

14 Giovanni BF, Giuseppe G: The clinical art and science of urine microscopy. Curr Opin Nephrol Hypertens 2003;12:625-632.

15 Praga M, González E: Acute interstitial nephritis: a mini review. Kidney Int 2010;77:956-961.

16 Mazur N, Greenberger PA, Regalado J: Clindamycin hypersensitivity appears to be rare. Ann Allergy Asthma Immunol 1999;82: 443-445.

17 Klainer A: Clindamycin. Med Clin North Am 1987;71:1169-1174. 